\title{
6 Hitzewellen: neue Herausforderungen für die medizinische Versorgung von älteren Menschen
}

\author{
Clemens Becker, Jochen Klenk, Julia Frankenhauser-Mannuß, \\ Ulrich Lindemann und Kilian Rapp
}

C. Günster ||. Klauber | B.P. Robra | C. Schmuker | A. Schneider (Hrsg.) Versorgungs-Report Klima und Gesundheit. DOI 10.32745/9783954666270-6, @ MWV Medizinisch Wissenschaftliche Verlagsgesellschaft Berlin 2021

Die zunehmende Anzahl an Hitzewellen in den letzten Jahren mit mehreren 10.000 Toten allein in Deutschland stellt eine erhebliche Bedrohung dar. Ältere Menschen sind hier besonders betroffen. Trotz Vorliegen guter Hitze-Aktionspläne ist die Umsetzung dieser Pläne unzureichend, möglicherweise wegen unklarer Zuständigkeiten und Priorisierung von Maßnahmen. Die Identifikation von Hoch-Risikopersonen im Pflegeheim und bei zuhause lebenden Personen durch ein einfaches Screening-Instrument könnte für Hausärzte die Initiierung von präventiven Maßnahmen erleichtern. Die Auswertung von Routinedaten der größten deutschen Krankenkasse für die Jahre 2008-2015 in BadenWürttemberg ergab, dass die Identifikation der Hoch-Risikopersonen über die Pflegestufe möglich ist. Bei Temperaturen über $30^{\circ} \mathrm{C}$ zeigte sich ein erhöhtes Mortalitätsrisiko in Pflegestufe 2 und 3 im Pflegeheim sowie in Pflegestufe 3 bei zuhause lebenden Älteren. Bei personeller Knappheit im Sommer könnten Hausärzte bei der Versorgung ihrer Hoch-Risikopersonen durch die im Katastrophenschutz tätigen Hilfsorganisationen unterstützt werden. Voraussetzung dafür wäre die Information der Hausärzte durch die Pflegekassen über die Pflegestufe der Patienten zur Erstellung von Hoch-Risikolisten und die Einstufung von extremer Hitze als Katastrophenfall. Für beide Voraussetzungen fehlen bisher die gesetzlichen Grundlagen.
The increasing number of heat waves during the last years with several 10,000 deaths in Germany alone is a substantial threat, especially for older persons. Although heataction-plans were developed, their implementation is insufficient, possibly due to unclear responsibilities and priorities. The identification of high-risk persons in nursing homes and in community-dwellers by a simple screening tool could help general practitioners to initiate preventive measures. Analyses of routine data of the greatest German health insurance company for the years 2008-2015 in Baden-Württemberg showed that the identification of high-risk persons is possible using care levels. An increased mortality risk at temperatures of $30^{\circ} \mathrm{C}$ and higher was documented for care levels 2 and 3 in nursing homes and for care level 3 for community-dwelling older persons. In the event of staff shortages in summer, general practitioners could be supported in the care of their high-risk patients by the aid organizations acting in the disaster control. This would require information of the general practitioners by the care insurance on the level of care of patients to create high-risk lists and the classification of extreme heat as a disaster. The legal basis for both prerequisites is still missing. 


\subsection{Hitzebedingte Mortalität in Deutschland}

Der Klimawandel ist eine Bedrohung für die Umwelt und die natürlichen Ressourcen und damit für die Menschen in vielen Regionen der Erde. Eine Folge davon ist die zunehmende Zahl an Hitzewellen während der Sommermonate. So traten bei Betrachtung der letzten 70 Jahre die meisten und in ihren Auswirkungen schwerwiegendsten Hitzewellen innerhalb der letzten 20 Jahre auf (Russo et al. 2015). Seit 2003 wurden alle 3-4 Jahre länger andauernde Hitzewellen beobachtet. In ganz Europa verstarben allein 2003 über 70.0oo Menschen an den Folgen extremer Hitze (Robine et al. 2008), davon etwa 7.600 in Deutschland (An der Heiden et al. 2019a). Von der Übersterblichkeit betroffen waren überwiegend alte gebrechliche Menschen. Weitere Hitzeperioden in Deutschland in den Jahren 2015 und 2018 haben dem Thema zudem Platz und Aufmerksamkeit verschafft. Im Bundesgesundheitsblatt beispielsweise wurde das Thema 2019 in einem Schwerpunktheft aufgegriffen. Obwohl sich während der letzten Dekade leicht reduzierte Mortalitätsraten andeuten (An der Heiden et al. 2020; Todd u. Valleron 2015), zeigt sich, dass es bislang nicht gelungen ist, eine durchgängige wirksame Strategie zu entwickeln und vermeidbare Todesfälle, vor allem bei älteren Menschen, zu reduzieren. Obwohl für Deutschland insgesamt bisher (Februar 2021) noch keine Zahlen veröffentlicht wurden, gehen Schätzungen auf der Grundlage der Zahlen einzelner Bundesländer (An der Heiden 2019b) von etwa 8.00o Hitzetoten im Jahr 2018 aus. Dies wäre mehr als im Katastrophenjahr 2003 und mehr als doppelt so viel wie die Anzahl der Verkehrstoten im gleichen Jahr (Statistisches Bundesamt 2019). Das Fazit der meisten Experten lautet, dass zwar sehr gute Hitzeaktionspläne entwickelt wurden (Bund/Länder Ad-hoc Arbeitsgruppe Gesundheitliche Anpassung an die Folgen des Klimawandels 2017), ein angemessenes Warnsystem (Deutscher Wetterdienst) vorliegt und ein Monitoring der Landesgesundheitsämter durchgeführt wird, dass aber die Umsetzung der Maßnahmen bei den Akteuren vor Ort noch unzureichend erfolgt (Capellaro u. Sturm 2015). Zu den Akteuren und Institutionen zählen an erster Stelle Ärzte, Krankenhäuser, Pflegedienste, Pflegeheime, Freiwilligendienste und (pflegende) Angehörige. Dabei ist unklar, welche Maßnahmen und Zuständigkeiten wie getriggert werden sollen. Problematisch ist auch, dass Hitzeextreme nicht selten zur Haupturlaubszeit auftreten, wenn die Personaldecke der Akteure im Gesundheitswesen reduziert ist.

Aus internationalen Studien sind Risikofaktoren für eine erhöhte Vulnerabilität von Risikopersonen bei Hitzewellen bekannt. Die wichtigsten Faktoren sind (Herrmann et al. 2019):

- höheres Alter (überwiegend 75+ Jahre)

- alleinlebende Personen

- höhere Wohnetagen (aufgrund höherer Hitzebelastung)

- niedriger Sozialstatus

n Immobilität

Folgende Krankheiten und Medikamente sind zudem mit einem erhöhten Risiko bei Hitzebelastung verbunden (Herrmann et al. 2019):

- Demenz und Depression

- Koronare Herzkrankheit und Herzinsuffizienz

- pulmonale Erkrankungen wie die COPD

- chronische Nierenkrankheiten

- Suchterkrankungen

- Einnahme von Sedativa

- transdermal und subkutan verabreichte Arzneimittel (wie opiathaltige Schmerzmittel oder Insulin)

Weder für den ambulanten noch für den stationären Bereich stehen bisher Screening-Instrumente zur Erfassung gefährdeter Personen zur Verfügung. IT-basierte Funktionen einer Praxissoftware könnten hier hilfreich sein. Es ist daher wichtig, nach pragmatischen Lösungsansätzen zu suchen. Ein Ansatz ist die Identifikation der Risikopersonen über das Setting und/oder die Pflegestufe bzw. den Pflegegrad. 


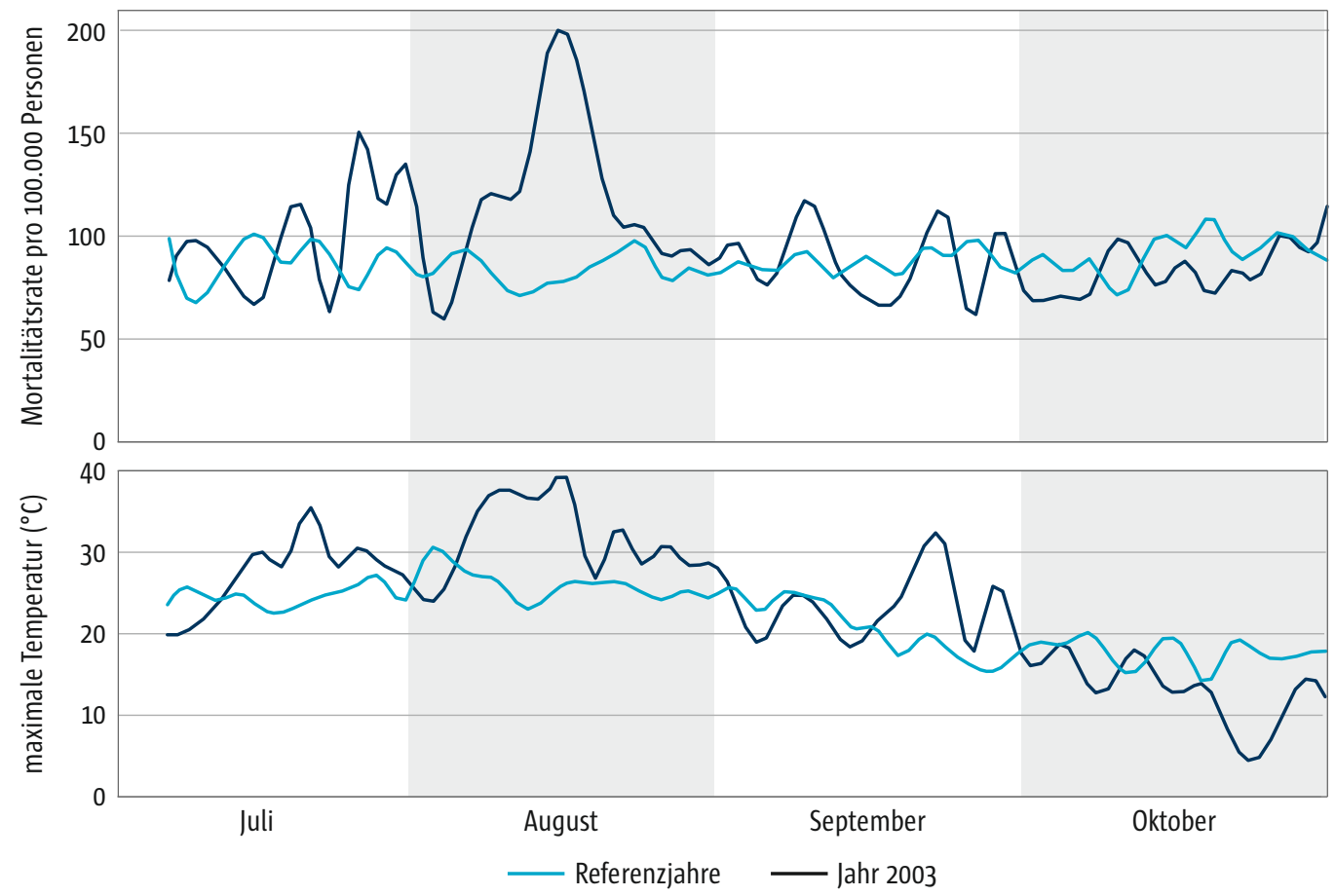

Abb. 1 Maximaltemperatur und Mortalität im Jahr 2003 und den Referenzjahren 2001, 2002, 2004 und 2005 von Juli bis Oktober bei Pflegeheimbewohnern in Baden-Württemberg (nach Klenk et al. 2010)

\subsection{Analysen mit Routinedaten der AOK Baden-Württemberg}

Vor 10 Jahren konnte anhand von Daten der AOK-Versicherten in Baden-Württemberg gezeigt werden, dass die hitzebedingte Mortalität bei Pflegeheimbewohnern im Jahr 2003 im Vergleich zu den Jahren davor und danach sehr hoch war (Klenk et al. 2010). In Abbildung 1 ist die erhebliche Übersterblichkeit während der Hitzewelle in den ersten zwei Augustwochen des Jahres 2003 gut zu erkennen. Dabei zeigte sich, dass nicht nur die absolute Sterblichkeit, sondern auch die hitzebedingte Übersterblichkeit mit zunehmender Gebrechlichkeit, d.h. ansteigender Pflegestufe, zunahm.

Wenn Pflegebedürftigkeit über das Merkmal Pflegestufe bzw. Pflegegrad definiert wird, dann lebt der überwiegende Teil pflegebedürftiger Menschen in Deutschland zuhause. Viele dieser Personen verfügen anders als Pflegeheimbewohner während Hitzewellen über keine oder über eine zeitlich limitierte personelle Unterstützung. Es stellte sich daher die Frage, inwieweit diese spezifische Personengruppe besonders vulnerabel gegenüber Hitzewellen ist. $\mathrm{Zu}$ dieser Frage lagen bisher keine Studien vor.

In einem vom Bundesministerium für Gesundheit geförderten Projekt (BMC 415-431641/687; Becker et al. 2019; Herrmann et al. 2019) wurde daher untersucht, a) ob Pflegestufen als Surrogat-Marker für eine besonders hohe Vulnerabilität gegenüber Hitzebelastung geeignet sein könnten, und b) ob pflegebedürftige Menschen, die zuhause leben, ein besonderes Risiko haben. Ziel war es, mit den Ergebnissen Pflegedienste, Pflegeheime und niedergelassene Ärzte darin zu unterstützen, besonders hitzegefährdete Personen zu erkennen. Für diese Untersuchung wurden Routinedaten der AOK 
II Gesundheitliche Auswirkungen des Klimawandels und Herausforderungen für die medizinische Versorgung in Deutschland

Tab. 1 Alters- und geschlechtsadjustierte tägliche Mortalität/100.000 Personentage bei rund 390.000 Versicherten über 65 Jahren der AOK Baden-Württemberg, die mit Pflegestufen 1 bis 3 entweder im Pflegeheim oder zu Hause leben, bezogen auf die maximale Tagestemperatur, 2008-2015

\begin{tabular}{|c|c|c|c|c|c|c|}
\hline \multirow{3}{*}{$\begin{array}{l}\text { Maximale } \\
\text { Temperatur }\end{array}$} & \multicolumn{3}{|c|}{ Pflegeheim } & \multicolumn{3}{|c|}{ zuhause lebend } \\
\hline & \multicolumn{3}{|c|}{ Pflegestufe } & \multicolumn{3}{|c|}{ Pflegestufe } \\
\hline & 1 & 2 & 3 & 1 & 2 & 3 \\
\hline $0^{\circ} \mathrm{C}$ & $\begin{array}{l}82,35 \\
(79,24-85,58)\end{array}$ & $\begin{array}{l}155,27 \\
(150,98-159,68)\end{array}$ & $\begin{array}{l}219,01 \\
(211,64-226,64)\end{array}$ & $\begin{array}{l}30,67 \\
(29,45-31,94)\end{array}$ & $\begin{array}{l}99,15 \\
(96,08-102,33)\end{array}$ & $\begin{array}{l}257,31 \\
(248,80-266,11)\end{array}$ \\
\hline $5^{\circ} \mathrm{C}$ & $\begin{array}{l}82,26 \\
(80,29-84,27)\end{array}$ & $\begin{array}{l}147,99 \\
(145,16-150,87)\end{array}$ & $\begin{array}{l}212,07 \\
(207,50-216,73)\end{array}$ & $\begin{array}{l}30,10 \\
(29,26-30,97)\end{array}$ & $\begin{array}{l}93,80 \\
(91,67-95,99)\end{array}$ & $\begin{array}{l}252,63 \\
(247,37-258,01)\end{array}$ \\
\hline $10^{\circ} \mathrm{C}$ & $\begin{array}{l}78,37 \\
(76,64-80,13)\end{array}$ & $\begin{array}{l}143,88 \\
(141,43-146,38)\end{array}$ & $\begin{array}{l}204,05 \\
(200,03-208,16)\end{array}$ & $\begin{array}{l}28,66 \\
(27,94-29,40)\end{array}$ & $\begin{array}{l}91,48 \\
(89,63-93,36)\end{array}$ & $\begin{array}{l}243,61 \\
(238,97-248,35)\end{array}$ \\
\hline $15^{\circ} \mathrm{C}$ & $\begin{array}{l}71,83 \\
(70,23-73,48)\end{array}$ & $\begin{array}{l}132,98 \\
(130,64-135,36)\end{array}$ & $\begin{array}{l}193,31 \\
(189,47-197,22)\end{array}$ & $\begin{array}{l}25,71 \\
(25,03-26,41)\end{array}$ & $\begin{array}{l}83,46 \\
(81,67-85,30)\end{array}$ & $\begin{array}{l}236,50 \\
(232,00-241,08)\end{array}$ \\
\hline $20^{\circ} \mathrm{C}$ & $\begin{array}{l}67,94 \\
(66,44-69,47)\end{array}$ & $\begin{array}{l}126,69 \\
(124,53-128,89)\end{array}$ & $\begin{array}{l}184,26 \\
(180,69-187,91)\end{array}$ & $\begin{array}{l}24,62 \\
(23,99-25,27)\end{array}$ & $\begin{array}{l}81,92 \\
(80,22-83,66)\end{array}$ & $\begin{array}{l}230,24 \\
(225,98-234,59)\end{array}$ \\
\hline $25^{\circ} \mathrm{C}$ & $\begin{array}{l}68,13 \\
(66,36-69,95)\end{array}$ & $\begin{array}{l}128,08 \\
(125,52-130,70)\end{array}$ & $\begin{array}{l}184,80 \\
(180,58-189,11)\end{array}$ & $\begin{array}{l}25,32 \\
(24,55-26,10)\end{array}$ & $\begin{array}{l}81,59 \\
(79,56-83,66)\end{array}$ & $\begin{array}{l}232,24 \\
(227,16-237,43)\end{array}$ \\
\hline $30^{\circ} \mathrm{C}$ & $\begin{array}{l}70,61 \\
(67,82-73,52)\end{array}$ & $\begin{array}{l}138,38 \\
(134,31-142,57)\end{array}$ & $\begin{array}{l}199,71 \\
(192,99-206,67)\end{array}$ & $\begin{array}{l}26,37 \\
(25,17-27,61)\end{array}$ & $\begin{array}{l}86,79 \\
(83,51-90,20)\end{array}$ & $\begin{array}{l}245,98 \\
(237,98-254,24)\end{array}$ \\
\hline $35^{\circ} \mathrm{C}$ & $\begin{array}{l}73,90 \\
(67,83-80,51)\end{array}$ & $\begin{array}{l}151,46 \\
(141,89-161,67)\end{array}$ & $\begin{array}{l}221,61 \\
(206,20-238,17)\end{array}$ & $\begin{array}{l}24,69 \\
(22,30-27,34)\end{array}$ & $\begin{array}{l}81,09 \\
(74,58-88,18)\end{array}$ & $\begin{array}{l}263,94 \\
(246,21-282,96)\end{array}$ \\
\hline
\end{tabular}

Baden-Württemberg der Jahre 2008-2015 genutzt. In Baden-Württemberg mit einer Bevölkerung von 10,4 Millionen Menschen sind etwa $20 \%$ der Bevölkerung 65 Jahre und älter. Die AOK hat in Baden-Württemberg etwa 4,5 Millionen Versicherte. Im Beobachtungszeitraum von 7 Jahren wurden Daten von fast 390.ooo Personen im Alter von über 65 Jahren mit mehr als 2 Millionen Personenjahren ausgewertet. Frauen waren in beiden untersuchten Personengruppen deutlich häufiger vertreten als Männer (Pflegeheim 76\%; zuhause lebend 67\%). Untersucht wurde der Einfluss der Außentemperatur (Deutscher Wetterdienst, WESTE) auf die tägliche Sterblichkeit in Abhängigkeit vom Setting (zuhause lebend oder im Pflegeheim) und der Pflegestufe. Die Mortalitätsraten mit 95\% Konfidenzintervallen wurden mithilfe eines Poisson-Modells mit Thin Plate Splines geschätzt, um auch nicht-lineare Zusammenhänge modellieren zu können. Die Analysen wurden für Alter und Geschlecht adjustiert, um eine mögliche Verzerrung durch diese Faktoren auf die eigentliche Fragestellung zu eliminieren.

Tabelle 1 zeigt die Mortalität in Abhängigkeit von der maximalen Tagestemperatur aufgeteilt nach Setting (zuhause lebend oder im Pflegeheim) und Pflegestufe. Unabhängig von der Temperatur fällt grundsätzlich auf, dass sich die Mortalität bei gleicher Pflegestufe zwischen zuhause lebenden und institutionalisierten Personen deutlich unterscheidet. Während bei Pflegestufe 1 und 2 im Pflegeheim höhere Mortalitätsraten beobachtet wurden, lagen bei Pflegestufe 3 die Mortalitätsraten bei zuhause lebenden Personen höher. Der Grund für die deutlichen Mortalitätsdifferenzen bei gleicher Pflegestufe kann aus den vorliegenden Daten 


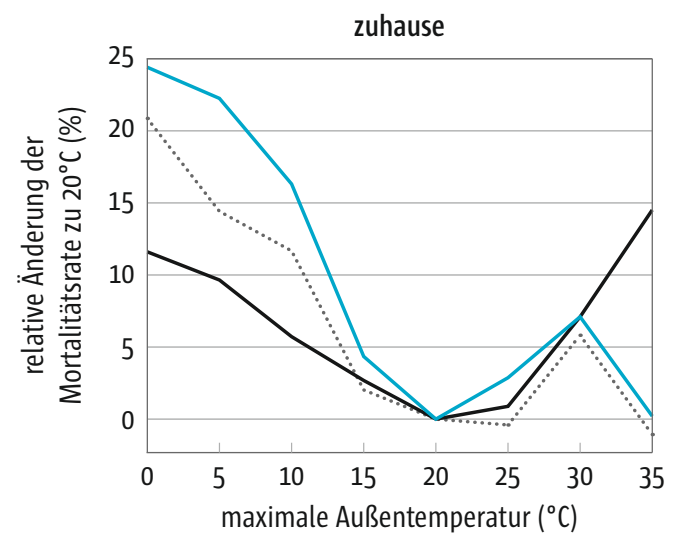

Pflegestufe:

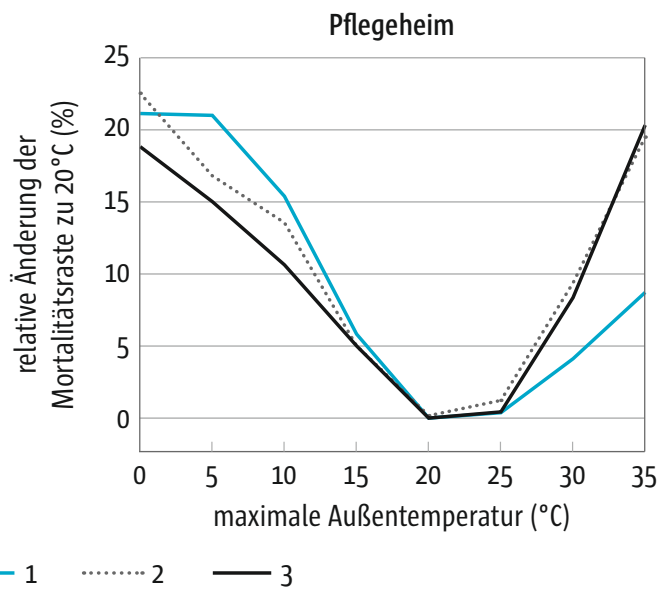

Abb. 2 Relative Veränderung der Mortalität in Abhängigkeit von der täglichen Maximaltemperatur im Vergleich zu einer Referenztemperatur von $20^{\circ} \mathrm{C}$ bei zuhause (links) oder im Pflegeheim (rechts) lebenden Versicherten der AOK Baden-Württemberg mit Pflegestufe 1, 2 oder 3 in den Jahren 2008-2015

nicht analysiert werden, dürfte aber am ehesten in einem unterschiedlichen Morbiditätsspektrum begründet liegen. In allen Personengruppen findet sich aber eine minimale Mortalität bei einer maximalen Außentemperatur von etwa $20^{\circ} \mathrm{C}$. Deshalb wurde diese Temperaturkategorie als Referenzwert genutzt, um die relative Veränderung der Mortalität in Abhängigkeit von der täglichen Maximaltemperatur bei zuhause oder im Pflegeheim lebenden Versicherten mit Pflegestufe 1, 2 oder 3 zu analysieren und in Abbildung 2 darzustellen. Hier findet sich ein relevanter Anstieg der Mortalität in allen Gruppen erst in der $30^{\circ} \mathrm{C}$-Temperaturkategorie. Eine erhebliche Zunahme der relativen Mortalität findet sich in der höchsten $35^{\circ} \mathrm{C}$ Temperaturkategorie v.a. in Pflegestufe 2 und 3 im Pflegeheim sowie in Pflegestufe 3 bei zuhause lebenden Personen, während dies für Pflegestufe 1 und 2 bei zuhause lebenden Personen nicht zu beobachten war.

Die Ursache der ansteigenden Mortalität bei niedrigen Temperaturen ist anhand unserer Datenbasis nicht zu beantworten und deshalb spekulativ. Es liegt aber nahe, dass dies u.a. auf Infektionskrankheiten in den Wintermonaten zurückgeführt werden kann (s. Kap. 3).

\subsection{Maßnahmen und Ausblick}

Die Vulnerabilität gegenüber Hitzewellen ist meist Folge einer eingeschränkten körperlichen Funktion und ungünstiger Kontextfaktoren des räumlichen und sozialen Umfelds (Lindemann et al. 2017; Lindemann et al. 2018). Im Wissen, dass es auch berufliche Risikogruppen (Landwirtschaft, Soldaten) und Säuglinge/ Kleinkinder gibt, die von Hitzewellen nachteilig betroffen sind, ist es offenkundig, dass chronisch kranke ältere Menschen die mit Abstand größte Risikogruppe sind. Die mit Daten der AOK Baden-Württemberg gewonnenen Ergebnisse zeigen, dass die Identifikation dieser Personen über die Pflegestufe einfach und zielführend ist. Man kann davon ausgehen, dass sich dies auch auf die neue Kategorisierung nach Pflegegraden übertragen lässt. Insgesamt bestätigen die Analysen frühere Untersuchungen aus Frankreich, die sich auf den Hitze-Sommer 2003 beziehen (Belmin et al. 2007). Auch hier war das Mortalitätsrisiko mit dem Grad der Abhängigkeit assoziiert.

In den letzten Jahren wurde die Wissensbasis für Ärzte, Pflege und medizinische Fachangestellte zu Maßnahmen bei extremer Hitze 
II Gesundheitliche Auswirkungen des Klimawandels und Herausforderungen

für die medizinische Versorgung in Deutschland

deutlich verbessert. Es existieren Blaupausen für die Schulung und Weiterbildung von Ärzten und medizinischen Fachangestellten (Klinikum Uni-München) und für Pflegeheime (Krampen 2020). Die Umsetzung solcher Maßnahmen bei Hausärzten wurde analysiert. Dabei wurden Möglichkeiten zur Verbesserung der Umsetzung aufgezeigt (Herrmann $u$. Sauerborn 2018).

Wie oben dargestellt, sind Personen mit Pflegebedarf und chronischen Erkrankungen die Gruppe mit der höchsten Gefährdung. Die wichtigsten Akteure zur Vermeidung von hitzebedingten Risiken dieser Personengruppen sind Hausärzte, Pflegedienste, Angehörige, ehrenamtliche Helfer, die Nachbarschaft und Mitarbeiter von Pflegeheimen.

Aufgrund ihres regelmäßigen Kontakts zu vulnerablen Personengruppen und deren Angehörigen und als Vertrauensperson im Gesundheitssektor nehmen Hausärzte hier eine zentrale Rolle ein. Zur Prävention hitzebedingter Risiken wurden vier hausärztliche Handlungsfelder identifiziert (Herrmann et al. 2019):

1. Kommunikation von Risiken und Präventionsmaßnahmen mit den Betroffenen und deren Familien (Konsultation und Broschüren)

2. Anpassung der Medikation (z.B. vorsommerlicher Medikamenten-Review mit Anpassung der Medikamente im Falle einer Hitzewelle)

3. Anpassung von Praxisabläufen (z.B. Kühlung der Praxisräume, Angebot von Getränken)

4. proaktive Kontaktaufnahme (z.B. Hausbesuche und Initiierung des Netzwerks der Helfer)

Für einen strukturierten Handlungsansatz fehlen allerdings bisher u.a. entsprechende Abrechnungsziffern. Ein solcher und wichtiger Ansatz ist das Erstellen von Listen der Risikopersonen, z.B. definiert über den Pflegegrad, in der elektronischen Gesundheitsakte. Dies ist zur Bündelung der personellen Ressourcen wichtig. Dazu wäre es aufgrund unserer Ergebnisse als erstem Schritt hilfreich, wenn den Hausärzten die Information über eine mögliche Pflegestufen- bzw. seit 2017 Pflegegrad-Einstufung zugänglich wäre. Hierfür muss allerdings erst eine gesetzliche Grundlage für den Datenabgleich geschaffen werden. Dies ist auch für ePA- und eGK-Ansätze denkbar. Die Liste wäre möglicherweise auch in anderen Situationen mit deutlich eingegrenzter Lokalisierung wie Überschwemmungen, Stromausfall etc. eine Option. Auch hier ist der Wohnort potenziell hilfsbedürftiger Personen den Einsatzhelfern nicht systematisch bekannt. Mithilfe einer Risikoliste würde dem Hausarzt die Planung erleichtert, wer ärztliche Hilfe oder die Unterstützung durch medizinische Fachangestellte benötigt, oder wem schon dadurch geholfen ist, dass das soziale Netzwerk aktiviert wird.

Bei der ggf. erforderlichen Anpassung der Medikation sind die Reduktion von Diuretika, die Dosisanpassung der Blutdruckmedikamente, das Pausieren und Reduzieren von Anticholinergika (Bsp. zur Therapie einer Dranginkontinenz) und die Anwendung des Bundesmedikationsplans mit Anpassungsvorgaben zu prüfen (s. Kap. 4). Im Kontext der Medikation wären eine Verbesserung der Beipackzettel mit Hitze-relevanten Hinweisen (BfARM) und die Etablierung einer funktionierenden Beratung durch die Apotheker wünschenswert. Einige Arzneimittelnebenwirkungen sind weitgehend unbekannt wie das Risiko von Hypoglykämien durch verstärkte Hautdurchblutung bei subkutanen Insulingaben oder toxische Plasmaspiegel durch transdermale Schmerzpflaster (z.B. Fentanyl oder Buprenorphin).

Die Realität in der Versorgung sieht allerdings nach wie vor anders aus. Nur einzelne Arztpraxen verfügen über ein systematisches Krisenmanagement. Es gibt nur in Ausnahmefällen einen Medikations-Review im Frühsommer. Eine proaktive Kontaktaufnahme bei Höchstrisikopersonen durch den Arzt im Falle einer Hitzewelle findet bei personeller Knappheit oft nicht statt. Ebenfalls findet eine pro- 
aktive Beratung in Apotheken nur in Ausnahmefällen statt. Die ambulanten Pflegedienste berichten zudem über mangelnde Möglichkeiten der Kommunikation mit niedergelassenen Ärzten. Für subkutane Infusionen als eineTherapieoption bei mangelnder Flüssigkeitsaufnahme ist meist keine Behandlungsplanung vorhanden. Es bedarf deshalb verschiedener Anpassungen, um Hitzeaktionspläne für ältere Menschen in der Versorgung wirksam zu implementieren.

Es ist offenkundig, dass die Arbeitskapazitäten der Hausärzte begrenzt sind und Zeit für Screening, Assessment und Beratung kostbar ist. Daher ist es von entscheidender Bedeutung, dass mit den begrenzten Ressourcen die besonders gefährdeten Personen identifiziert und adressiert werden. Es wurden deshalb Strategien vorgeschlagen, wie bei Personalknappheit die in Deutschland gut organisierten Hilfsorganisationen mit ihren hauptamtlichen Akteuren in die ambulante Versorgung eingebunden werden könnten (Becker et al. 2019). Dazu müssten Hitzeextreme entsprechend dem von Deutschland ratifizierten Sendai UN-Rahmenwerk (Sendai Framework for Disaster Risk Reduction 2015-2030) als Katastrophenfall behandelt und bei extremer Hitze z.B. durch das Gesundheitsamt der Hitzenotstand ausgerufen werden, um Hilfsorganisationen in die Versorgung besonders gefährdeter Personen mit einzubeziehen. Eine Überprüfung solcher Konzepte und eine nachfolgend regulatorische Umsetzung auf Bundes-, Landes- oder kommunaler Ebene werden empfohlen (Mücke $u$. Litvinovitch 2020), sind allerdings bisher noch nicht erfolgt.

Die wichtigsten Maßnahmen wurden für die Fachöffentlichkeit in einem CME-Artikel 2019 zusammengefasst und inzwischen häufig abgerufen (Herrmann et al. 2019). Es wäre zu wünschen, dass die Fachgesellschaft der Allgemeinmedizin sich dem Thema ebenfalls verstärkt zuwendet.

\section{Literatur}

An der Heiden M, Muthers S, Niemann H, Buchholz U, Grabenhenrich L, Matzarakis A (2019a) [Estimation of heat-related deaths in Germany between 2001 and 2015]. Bundesgesundheitsblatt, Gesundheitsforschung, Gesundheitsschutz 62(5), 571579

An der Heiden M, Buchholz U, Uphoff H (2019b) Schätzung der Zahl hitzebedingter Sterbefälle und Betrachtung der ExzessMortalität; Berlin und Hessen, 2018. Epid Bull 23, 193-197

An der Heiden M, Muthers S, Niemann H, Buchholz U, Grabenhenrich L, Matzarakis A (2020) Hitzebedingte Mortalität - Eine Analyse der Auswirkungen von Hitzewellen in Deutschland von 1992-2017. Deutsches Ärzteblatt 117, 603-609

Becker C, Herrmann A, Haefeli WE, Rapp K., Lindemann U (2019) [New approaches in preventing health risks and excess mortality of older persons during extreme heat]. Bundesgesundheitsblatt, Gesundheitsforschung, Gesundheitsschutz 62(5), 565-570

Belmin I, Auffray IC, Berbezier C, Boirin P, Mercier S, de Reviers B, Golmard JL (2007) Level of dependency: a simple marker associated with mortality during the 2003 heatwave among French dependent elderly people living in the community or in institutions. Age Ageing 36(3), 298-303

Bund/Länder Ad-hoc Arbeitsgruppe Gesundheitliche Anpassung an die Folgen des Klimawandels (2017) Handlungsempfehlungen für die Erstellung von Hitzeaktionsplänen zum Schutz der menschlichen Gesundheit. Bundesgesundheitsblatt, Gesundheitsforschung, Gesundheitsschutz 60(6), 662-672

Capellaro M, Sturm D (2015) Evaluation von Informationssystemen zu Klimawandel und Gesundheit. Umweltbundesamt, Dessau-Roßlau. URL: https://www.umweltbundesamt.de/ sites/default/files/medien/378/publikationen/umwelt_ und_gesundheit_03_2015_evaluation_von_informationssystemen_band_1_0.pdf (abgerufen am 18.02.2021)

Deutscher Wetterdienst. Wetter und Klima - Deutscher Wetterdienst - DWD-Apps. URL: https://www.dwd.de/DE/service/ dwd-apps/dwdapps_node.html (abgerufen am 18.02.2021)

Deutscher Wetterdienst. WEtterdaten und -STatistiken Express WESTE. URL: https://www.dwd.de/DE/klimaumwelt/cdc/ klinfo_systeme/weste/weste_node.html (abgerufen am 18.02.2021)

Herrmann A, Sauerborn R (2018) General practitioners' perceptions of heat health impacts on the elderly in the face of climate change-A qualitative study in Baden-Wurttemberg, Germany. Int J Environ Res Public Health, 15(5), 843

Herrmann A, Haefeli WE, Lindemann U, Rapp K, Roigk P, Becker C (2019) [Epidemiology and prevention of heat-related adverse health effects on elderly people]. Zeitschr Gerontol Geriatr 52(5), 487-502

Klenk J, Becker C, Rapp K (2010) Heat-related mortality in residents of nursing homes. Age Ageing 39(2), 245-252

Krampen R (2020) Klimaextreme - Handlungsempfehlungen für Pflegeheime und deren ordnungsrechtliche Überprüfung am Beispiel Hessen. Public Health Forum 28(1), 37-39 
Klinikum Uni-München. Materialien Hitze \& Gesundheit. URL: http://www.klinikum.uni-muenchen.de/BildungsmoduleAerzte/de/bildungsmodule-mfa/Materialien-Hitze-Gesundheit/index.html (abgerufen am 18.02.2021)

Lindemann U, Stotz A, Beyer N, Oksa |, Skelton DA, Becker C, Rapp K, Klenk I (2017) Effect of Indoor Temperature on Physical Performance in Older Adults during Days with Normal Temperature and Heat Waves. Int I Environ Res Public Health 14(2), 186

Lindemann U, Skelton DA, Oksa I, Beyer N, Rapp K, Becker C, Klenk I (2018) Social participation and heat-related behavior in older adults during heat waves and on other days. $Z$ Gerontol Geriatr 51(5), 543-549

Mücke HG, Litvinovitch JM (2020) Heat Extremes, Public Health Impacts, and Adaptation Policy in Germany. Int I Environ Res Public Health 17(21), E7862
Robine JM, Cheung SLK, Le Roy S, Van Oyen H, Griffiths C, Michel IP, Herrmann FR (2008) Death toll exceeded 70,000 in Europe during the summer of 2003. Comptes Rendus Biologies 331(2), 171-178

Russo S, Sillmann J, Fischer EM (2015) Top ten European heatwaves since 1950 and their occurrence in the coming decades. Environmental Research Letters 10(12), 124003

Sendai Framework for Disaster Risk Reduction 2015-2030. URL: https://www.undrr.org/publication/sendai-framework-disaster-risk-reduction-2015-2030 (abgerufen am 18.02.2021)

Statistisches Bundesamt (2019) Verkehrsunfälle. URL: https:// www.destatis.de/DE/Themen/Gesellschaft-Umwelt/Verkehrsunfaelle/_inhalt.html (abgerufen am 18.02.2021)

Todd N, Valleron AJ (2015) Space-Time Covariation of Mortality with Temperature: A Systematic Study of Deaths in France, 19682009. Environmental Health Perspectives 123(7), 659-664
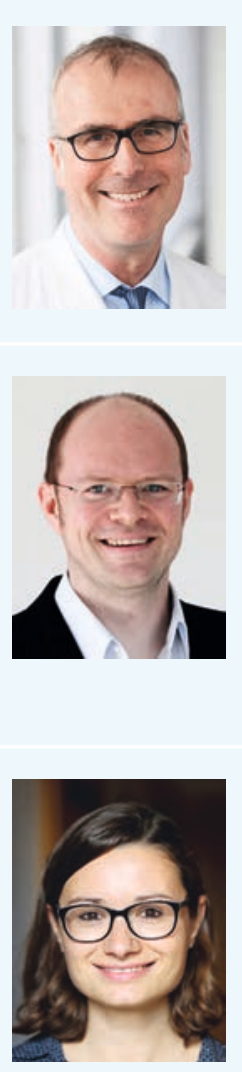

\section{Prof. Dr. Clemens Becker}

Chefarzt der Abteilung Geriatrie und Geriatrische Rehabilitation am Robert-Bosch-Krankenhaus Stuttgart. Er ist Professor für Gesundheits- und Bewegungswissenschaften an der Universität Stuttgart und unterrichtet Geriatrie an der Universität Tübingen.

\section{Prof. Dr. Jochen Klenk, MPH}

Professor für Gesundheitswissenschaften an der IB Hochschule für Gesundheit und Soziales sowie Projekt- und Arbeitsgruppenleiter am Institut für Epidemiologie und Medizinische Biometrie der Universität Ulm und an der Klinik für Geriatrische Rehabilitation im Robert-Bosch-Krankenhaus Stuttgart. Nach seinem Abschluss als Diplomingenieur für Medizintechnik an der Fachhochschule Ulm im Jahr 2000 sowie dem Masterabschluss in Public Health an der Universität in Ulm 2004 promovierte er 2009 in Humanbiologie an der Universität Ulm und habilitierte dort 2017. Seine Forschungsschwerpunkte sind erfolgreiches Altern, sensorbasierte Messung körperlicher Aktivität und Funktion sowie die Modellierung von Sturzrisikofaktoren.

\section{Dr. Julia Frankenhauser-Mannuß}

Studium der Soziologie mit Schwerpunkt Medizinsoziologie an der Universität Heidelberg. Seit 2009 ist sie bei der AOK Baden-Württemberg in unterschiedlichen Bereichen tätig und leitet dort aktuell Projekte aus dem Bereich der Rehabilitations- und Pflegeforschung. Berufsbegleitend promovierte sie von 2011-2015 am Universitätsklinikum Heidelberg in der Abteilung Allgemeinmedizin und Versorgungsforschung. 


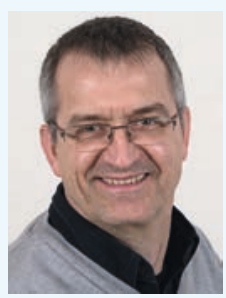

\section{Dr. Ulrich Lindemann}

Seit 1989 Diplom-Sportlehrer (DSHS Köln). Nach Tätigkeit als Trainer und Sporttherapeut promovierte er 2004 an der Universität Ulm und arbeitet seitdem als wissenschaftlicher Angestellter in der Klinik für Geriatrische Rehabilitation am Robert-Bosch-Krankenhaus in Stuttgart. Seine Forschungsschwerpunkte sind das Training und die Messung der körperlichen Leistungsfähigkeit und Aktivität älterer Menschen sowie modifizierende Faktoren.

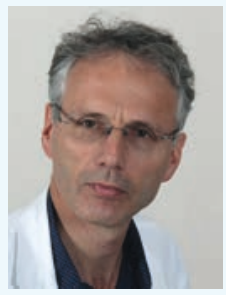

\section{Prof. Dr. Kilian Rapp, MPH}

Studium der Humanmedizin in Tübingen und Berlin. Facharztausbildung für Innere Medizin am Robert-Bosch-Krankenhaus Stuttgart und Zusatzbezeichnung Geriatrie. Aufbaustudiengang Public Health in Ulm. Wissenschaftliche Ausbildung am Institut für Epidemiologie und Medizinische Biometrie der Universität Ulm. Seit 2010 Oberarzt und stellvertretender Forschungsleiter in der Klinik für Geriatrische Rehabilitation des Robert-Bosch-Krankenhauses Stuttgart. Apl-Professur an der Universität Ulm. 